\title{
Von der Finanzkrise zu Depression und Deflation
}

Die Subprime-Immobilienkrise, die im Jahre 2007 offen ausbrach und 2008 eskalierte, ist in der Geschichte der Finanzmärkte kein Einzelfall. Die Krise folgt jenseits historischer Spezifika einem Muster, das sowohl theoretisch wie auch historisch gut durchleuchtet ist. In diesem Beitrag geht es um die Frage, ob und wie sich die Finanzmarktkrise auf ökonomisches Wachstum sowie das Güterpreisniveau auswirken wird. Im ersten Abschnitt wird auf die staatlichen Interventionen nach der Eskalation der Krise im Herbst 2008 eingegangen. Danach wird die Gefahr einer „großen Depression“ mit der Möglichkeit einer Deflation diskutiert. Abschließend werden Politiken gegen eine Depression aufgezeigt.

\section{STABILISIERUNG DES FINANZSYSTEMS}

Insgesamt haben Zentralbanken und Regierungen die richtigen Politiken zur Stabilisierung der strauchelnden Finanzsysteme betrieben. Sie wird gelingen.

Zentralbanken übernehmen weltweit ihre Funktion als Lender of Last Resort und stellen in unbegrenzter Höhe Liquidität zur Verfügung. Neben Geschäftsbanken wurden weitere Finanzinstitutionen, etwa Investmentbanken, unter die Fittiche der Zentralbank genommen. Eine Liquiditätsklemme im Finanzsystem ist somit eher unwahrscheinlich. Die öffentlichen Haushalte geben Garantien und Eigenkapitalspritzen an Finanzinstitutionen und helfen damit Instituten mit zu geringem Eigenkapital aufgrund fauler Kredite und sinkender Vermögensmarktpreise. Die Interventionen der öffentlichen Haushalte werden teuer. Es ist zu hoffen, dass der Staat bei seinen Eingriffen nicht einfach Geld gibt, sondern Eigentümerfunktionen mit allen Rechten eines Eigentümers einfordert. Dies scheint der fiskalisch günstigste Weg zu sein. Auch ordnungspolitisch ist staatliches Eigentum an Finanzinstituten unumgänglich, da sich das Finanzsystem selbst in die gegenwärtige Lage gebracht hat und aus eigener Kraft keinen Ausweg findet.

Die Finanzmarktkrise wirft die Frage nach sozialer Gerechtigkeit auf, da vor der
Krise exorbitante Einkommen in der Finanzbranche verdient wurden und die Kosten der Krise sozialisiert werden. Eine stärkere Besteuerung hoher Einkommen und Vermögen scheint zur Abmilderung sozialer Verwerfungen unumgänglich. Willem Buiter schlägt vor, dass der Staat bei Institutionen, die ohne Hilfen nicht überleben können, die Entscheidungsgewalt übernimmt. Der Staat könnte dann Manager entlassen, ihre Entlohnung und Abfindung bestimmen, Teile der Institute verkaufen und Geschäfte solcher Institute mit Steuerparadiesen und Offshore-Zentren untersagen. Die Bankenaufsicht BaFin oder die Bundesbank könnten in diesem Fall die Vertretung des Staates wahrnehmen. ${ }^{1}$

\section{DIE GEFAHR EINER "GROSSEN DEPRESSION"}

Gutes Anschauungsmaterial für den Aufbau einer Vermögensmarktblase und die Folgen der dann zwingend auftretenden Vermögensmarktdeflation stellt unter anderem die Weltwirtschaftskrise in den 1930er Jahren dar; jedoch auch Japan in den 1990er Jahren nach dem Ende der Aktien- und Immobilienblase, die sich in der zweiten Hälfte der 1980er Jahre entwickelte, kann als Lehrstück gelten. Gemeinsam ist Vermögensmarktdeflationen, dass sie zum Auftreten von Liquiditäts- und Solvenzproblemen führen, die als Ergebnis eine drastische Verknappung von Krediten an den Privatsektor nach sich ziehen. Joseph Stiglitz bekam unter anderm seinen Nobelpreis dafür, dass er verdeutlichte, dass auf Finanzmärkten immer eine Kreditrationierung stattfindet, denn Gläubiger werden aufgrund asymmetrischer Informationen nicht jedem Kreditnachfrager einen Kredit geben. Selbst eine Nullzinspolitik, wie in Japan ab Mitte der 1990er Jahre, kann dann keine Kreditexpansion der Banken erzwingen. Verschärfend kommt hin$\mathrm{zu}$, dass sinkende Immobilien- und Aktienpreise das Vermögen der Schuldner, das bei einem Kreditausfall den Gläubigern zur Verfügung steht, reduzieren. In der gegenwärtigen Situation scheint die Gefahr immens, dass aufgrund der Einschätzung der zukünftigen Entwicklung seitens der Ban- ken, des geschrumpften Eigenkapitals des Finanzsystems und schlechter werdender Sicherheiten der Schuldner eine scharfe Kreditrationierung gegenüber dem Privatsektor und damit eine scharfe Rezession in vielen Industrieländern nicht zu verhindern ist. Zwar kann durch staatliche Kapitalspritzen das Eigenkapitalproblem der Finanzinstitutionen entschärft werden, jedoch wird dadurch eine Kreditrationierung nicht zwingend verhindert. Auch ein staatliches Management der Banken wird deren Zurückhaltung bei der Kreditvergabe nicht unmittelbar verändern können. ${ }^{2}$

Eine Reduzierung des Kreditvolumens kommt nicht nur von der Angebotsseite. Denn Schuldner - ob Unternehmen oder private Haushalte - werden bei ihrer Kreditaufnahme vorsichtiger, da auch sie in einem unsichereren Umfeld agieren müssen. Zudem mussten Unternehmen und Haushalte aufgrund fallender Aktien- und Immobilienpreise hohe Vermögensverluste hinnehmen. Dieser Vermögenseffekt dämpft nicht nur die Bereitschaft zur Kreditaufnahme, sondern auch die Konsumund Investitionsnachfrage.

Zusammenfassend kann man feststellen, dass sowohl theoretische Argumente als auch historische Erfahrungen mit Finanzmarktkrisen eine massive Schrumpfung der aggregieren Nachfrage und eine unter Umständen scharfe Rezession mit hohen Beschäftigungsverlusten erwarten lassen. Ein Einbruch der Nachfrage hat die Tendenz, weitere Einkommens- und Pro-

\footnotetext{
1 Buiter, W.: Notfalls verstaatlichen, in: Die Zeit 42 vom 9. Oktober 2008

2 Vgl. zur Finanzmarktkrise Herr, H./Stachuletz, R. (2007): "New Fashion in Finance" und Finanzmarktstabilität, in: WSI Mitteilungen 12, S. 650656; Herr, H./Stachuletz, R. (2008): Deregulierung, Finanzmarktdesaster und Reformoptionen: Die Hoffnung stirbt zuletzt, in: Internationale Politik und Gesellschaft 3, S. 11-27.
}

Hansjörg Herr, Prof. Dr., Fachhochschule für Wirtschaft Berlin. Arbeitsschwerpunkte: Supranationale Integration, Entwicklungstheorie und -politik.

e-mail: hansherr@fhw-berlin.de 
duktionseinbußen nach sich zu ziehen. Die Spirale der Einkommens- und Beschäftigungsverluste dreht sich in der Folge weiter nach unten; der Marktmechanismus führt dann in eine immer tiefere Krise.

In diesem Szenario besteht eine weitere und sehr erstzunehmende Gefahr, nämlich die Möglichkeit einer Gütermarktdeflation. Ein fallendes Preisniveau wird durch zwei Faktoren angetrieben. Erstens durch eine zu geringe Güternachfrage, die Unternehmen neben einer Reduktion der Produktion zu einer Senkung der Preise zwingt. Eine Nachfragedeflation ist während eines Abschwungs unumgänglich und tritt regelmäßig auf. Gefährlich wird die Lage, wenn eine Nachfragedeflation durch eine Kostendeflation verstärkt wird, die den zweiten Faktor fallender Preise darstellt. Kostendeflationen finden ihre Hauptursache in fallenden Lohnstückkosten. Funktional sind aus diesem Grunde Lohnerhöhungen, die der trendmäßigen Erhöhung der Produktivität plus der Zielinflationsrate der Zentralbank entsprechen. Unterstellt man für Deutschland eine mittelfristige Produktivitätserhöhung von $1,5 \%$ und akzeptiert man die niedrige Zielinflationsrate der Europäischen Zentralbank (EZB) von knapp unter $2 \%$, dann müsste sich das Niveau der Geldlöhne in Deutschland jährlich um knapp unter 3,5 \% erhöhen. Erhöhen sich die Löhne unterhalb dieser Norm oder sinken gar, dann treten deflationäre Prozesse auf. Dass Deflationen kein Gespenst aus den 1930er Jahren sind, zeigt die Entwicklung in Japan ab Mitte der 1990er Jahre. Sinkende Geldlöhne reduzierten die Lohnstückkosten und führten zur Deflation. Der Deflationsprozess nahm zwar nicht wie in den 1930er Jahren einen kumulativen Charakter an, jedoch war er stark genug, Japan in eine Stagnation zu treiben, die bis heute nicht grundlegend überwunden ist. Auch in Deutschland war die Erhöhung des gesamtwirtschaftlichen Lohnniveaus zu niedrig, denn von Mitte der 1990er Jahre bis 2007 blieb das Niveau der Lohnstückkosten in Deutschland annähernd konstant. In einzelnen Jahren stand Deutschland am Rande einer deflationären Entwicklung. ${ }^{3}$

Gütermarktdeflationen haben für die ökonomische Entwicklung katastrophale Folgen, da sie die reale Schuldenlast aller Schuldner erhöhen. In einer Deflation sinken zwangsläufig die nominellen Zahlungszuflüsse der Schuldner, während die nominellen Zahlungen zur Schuldentilgung unverändert bleiben. Eine Deflation bei einem hohen Schuldenbestand führt unvermeidlich zu massenhaften finanziellen Zusammenbrüchen von Unternehmen und Privathaushalten. Die Kohärenz der Ökonomie zerbricht, da Zahlungsketten an allen Ecken und Enden der Ökonomie reißen. Irving Fisher hat bei seiner Analyse der 1930er Jahre zurecht betont, dass bei einer Deflation das ökonomische Boot nicht nur schwankt, sondern kentert. ${ }^{4}$ Es versteht sich von selbst, dass ein Deflationsprozess auch unabhängig von der Zerrüttung des Finanzsystems die Güternachfrage drastisch senkt. Welches Unternehmen wird heute eine Maschine kaufen, wenn es damit rechnet, dass sein Konkurrent die Maschine in der Zukunft günstiger kaufen kann? Welcher Haushalt kauft heute ein Auto, wenn es in der Zukunft billiger ist? Deflationen sind gefährlich, weil sie das volkswirtschaftliche System von Schuldnern und Gläubigern zerrütten und Güternachfrage und Produktion zum kumulativen Schrumpfen bringen.

Deflationsprozesse treten auf, wenn der nominelle Lohnanker reißt und die Geldlöhne sinken. Ein Lohnanker gegen Deflation basiert auf Arbeitsmarktinstitutionen einschließlich Lohnbildungssystemen, die Geldlohnsenkungen ausschließen und Lohnerhöhungen entsprechend der trendmäßigen Produktivitätsentwicklung plus der Zielinflationsrate der Zentralbank realisieren. Die Dramatik der gegenwärtigen ökonomischen Situation besteht darin, dass nicht nur die Finanzsysteme ab den 1980er Jahren weltweit dereguliert wurden, sondern auch die Arbeitsmärkte. Damit wurden die zwei wichtigsten Stabilitätssäulen in der Ökonomie ausgehöhlt. Nehmen wir Deutschland als Beispiel: Eine immer größere Anzahl von Beschäftigten arbeitet in prekären Arbeitsverhältnissen; ein koordinierter Lohnbildungsmechanismus funktioniert nur noch in einem Teil der Ökonomie; in einzelnen Branchen (etwa spezifischen Dienstleistungen), Regionen (etwa Ostdeutschland) und Unternehmenstypen (etwa Kleinunternehmen) sind Gewerkschaften und Arbeitgeberverbände so geschwächt, dass das hergebrachte System der Lohnverhandlungen nicht mehr funktioniert. Bei hoher Arbeitslosigkeit sind in der gegenwärtigen Lage Senkungen des Lohnniveaus nicht mehr auszuschließen. ${ }^{5}$ Die Begleitmusik der Deregulierungswellen in den Finanz- und Arbeitsmärkten lieferten das neoliberale Politikverständnis und der neoklassische Theorieansatz, die sich nach den Wahlsiegen von Ronald Reagan und Margret Thatcher in den 1980er Jahren durchsetzten und auf die meisten westlichen Länder übertrugen.

Führt die Finanzmarktkrise zu einer scharfen Rezession mit einem Anschwellen der Arbeitslosigkeit und reißt dann der Lohnanker, sind Entwicklungen wie in den 1930er Jahren wahrscheinlich.

\section{NACHFRAGESTABILISIERUNG UND STÄRKUNG DES LOHNANKERS}

Die Stabilisierung der Finanzmärkte nach dem Subprime-Debakel war zwar wichtig und richtig, jedoch ist dies nicht ausreichend. Es müssen gleichzeitig zwei weitere Problembereiche angegangen werden: die Stabilisierung der Güternachfrage und die Stärkung des Lohnankers.

Der Staat hat die Aufgabe, bei einer Schrumpfung der privaten Nachfrage die gesamtwirtschaftliche Nachfrage zu stabilisieren. Dazu muss er zunächst die automatischen Stabilisatoren wirken lassen. Das bedeutet, dass die Reduzierung der Steuereinnahmen aufgrund der sinkenden Wertschöpfung und die krisenbedingten Ausgabenerhöhungen, beispielsweise aufgrund steigender Arbeitslosigkeit, passiv hingenommen werden. Jedoch reicht diese passive antizyklische Politik des Staates nicht aus. Zusätzliche Stützungsmaßnahmen für die Konjunktur sind notwendig, wobei eine Erhöhung der staatlichen Ausgaben grundsätzlich einer Senkung von Steuern vorzuziehen ist. Unterstützt werden könnte eine solche Politik durch staatliche Ausgaben, die durch Steuererhöhungen bei hohen Einkommen und hohen Vermögen sowie die Austrocknung von Steueroasen finanziert werden.

Japan konnte nach dem Platzen seiner Aktien- und Immobilienblase Anfang der 1990er Jahre eine Entwicklung wie in den

3 Zur Deflation vgl. Heine, M./Herr, H. (2003): Volkswirtschaftslehre. Paradigmenorientierte Einführung in die Mikro- und Makroökonomie, München 2003; Heine, M./Herr, H. (2008): Die Europäische Zentralbank. Eine kritische Einführung in die Strategie und Politik der EZB und der Probleme der EWU, Marburg.

4 Fisher, I. (1933): The Debt-Deflation Theory of Great Depressions, in: Econometrica 1, S. 337-357.

5 Bosch, G./Kalina, T./Weinkopf, C. (2008): Niedriglohnbeschäftigte auf der Verliererseite, in: WSI Mitteilungen 8, S. 423-430. 
1930er Jahren nur durch die staatliche Stützung maroder Finanzinstitute und eine über viele Jahre hinweg expansive Fiskalpolitik verhindern; allerdings konnte trotz dieser Fiskalpolitik im Zusammenspiel mit einer Null-Zins-Politik der Bank of Japan kein Aufschwung erzwungen werden. Die Staatsverschuldung in Japan beträgt derzeit rund $200 \%$ am Bruttoinlandsprodukt (BIP) - der Preis der Vermögensmarktblase und der deflationären Entwicklung. Aber Japan hat Fehler gemacht. Lernen kann man von Japan, dass das Solvenzproblem von Finanzinstituten nicht zögerlich gelöst werden darf, dass Geld- und Fiskalpolitik schnell und substanziell auf eine Vermögensmarktdeflation reagieren müssen und dass eine Politik der Lohnsenkungen äußerst negative Folgewirkungen hat. ${ }^{6}$

Auf den Arbeitsmärkten sind Politiken notwendig, die ein Reißen des Lohnankers bei der zu erwartenden Erhöhung der Arbeitslosigkeit verhindern. In Deutschland ist schon aus diesem Grund die Einführung eines Mindestlohns wichtiger als je zuvor. Tarifverträge sollten, wie früher üblich, flächendeckend von staatlicher Seite für alle Unternehmen in einer Branche und einer Region als bindend erklärt werden. Das Tarifverhandlungssystem in Deutschland ist durch die Unterstützung von Gewerkschaften und Arbeitgeberverbänden zu stärken. Prekäre Arbeitsplätze sollten systematisch abgebaut werden.

Geldpolitik wird von der EZB für die Europäische Währungsunion (EWU) ge- macht. Auch die Bereiche Fiskalpolitik und Verhinderung des Reißens des Lohnankers sind auf EWU-Ebene anzugehen. Fiskalpolitik kann in Europa effektiv nur auf EWUEbene betrieben werden. Neben nationalen Konjunkturprogrammen bieten europaweit abgestimmte Infrastrukturinvestitionen im Bereich des Verkehrs oder der Ökologie Raum für stabilisierende Fiskalpolitik. Die EWU-Länder sollten sich auf eine gemeinsame Mindestlohnpolitik und andere arbeitsmarktpolitische Grundsätze einigen. Ideen einer europäischen Wirtschaftsregierung auf EWU-Ebene gehen in die richtige Richtung. Denn die EWU macht nur dann einen Sinn, wenn Bankenaufsicht, Fiskalpolitik einschließlich Steuerpolitik, Mindestlöhne, Tarifverhandlungssysteme, Mitbestimmung in Unternehmen, Industriepolitik etc. in Grundzügen auf EWU-Ebene abgestimmt werden. Dies schließt ein Europa der verschiedenen Geschwindigkeiten ein. Länder, die der EWU angehören, müssen bei ihren Integrationsbemühungen vorangehen, andere EU-Länder, die diesen Schritt nicht wollen, können ein niedrigeres Integrationsniveau wählen.

Das Globalisierungsprojekt der letzten Jahrzehnte muss überdacht werden. Es hat nicht nur zu regelmäßigen Aktien- und Immobilienblasen mit hohen Folgekosten geführt, sondern eine größere Instabilität vieler für die Weltwirtschaft relevanter Preise bewirkt. Wechselkurse, Rohstoffpreise oder Nahrungsmittelpreise sind zu Schockzentren der Weltwirtschaft geworden. Wäh- rungs- und Finanzkrisen haben seit den 1980er Jahren in den peripheren Ländern der Weltwirtschaft an Häufigkeit und Stärke zugenommen. Die derzeitige Finanzkrise birgt die große Gefahr, dass Länder wie die Ukraine, die Türkei, Vietnam, Südafrika, Ungarn oder die baltischen Länder eine neue Runde von peripheren Währungskrisen einläuten. Von einer effizienten Allokation der Ressourcen kann angesichts solcher Turbulenzen schwerlich gesprochen werden, allenfalls von gigantischer Verschwendung. Gleichzeitig kam es in nahezu allen Ländern der Welt zu einer zunehmenden Ungleichheit bei der Einkommens- und Vermögensverteilung und einem Anschwellen prekärer Arbeitsverhältnisse. Das Niveau der Unsicherheit hat sich für alle Wirtschaftssubjekte erhöht für Arbeitnehmer, Kleinunternehmer, Vermögenshaushalte, Finanzinstitutionen und selbst multinationale Unternehmen. Es ist überfällig, auf allen politischen Ebenen über neue Rahmenbedingungen des Wirtschaftens nachzudenken und zu einem Regulierungsrahmen zu kommen, der den Globalisierungsprozess in stabilere und gerechtere Bahnen lenkt.
Heine, M./Herr, H./Kaiser C. (2006): Wirtschaftspolitische Regime westlicher Industrieländer theoretische Analyse und Fallstudien über Japan, Großbritannien, die USA und Deutschland, BadenBaden 